PROCEEDINGS OF THE

AMERICAN MATHEMATICAL SOCIETY

Volume 128, Number 12, Pages 3547-3552

S 0002-9939(00)05473-3

Article electronically published on May 18, 2000

\title{
STRONGLY ASYMPTOTICALLY STABLE FROBENIUS-PERRON OPERATORS
}

\author{
RADU ZAHAROPOL
}

(Communicated by Dale Alspach)

Dedicated to Professor Alexandra Bellow in celebration of her achievements in all the aspects of being that involve mathematics

\begin{abstract}
Let $(X, \Sigma, \mu)$ be a $\sigma$-finite measure space and let $T: L^{1}(X, \Sigma, \mu) \rightarrow$ $L^{1}(X, \Sigma, \mu)$ be a Frobenius-Perron operator.

In 1997 Bartoszek and Brown proved that if $T$ overlaps supports and if there exists $h \in L^{1}(X, \Sigma, \mu), h>0$ on $X$, such that $T h=h$, then $T$ is (strongly) asymptotically stable.

In the note we prove that instead of assuming that $h>0$ on $X$, it is enough to assume that $h \geq 0$ and $h \neq 0$. More precisely, we prove that $T$ is asymptotically stable if and only if $T$ overlaps supports and there exists $h \in L^{1}(X, \Sigma, \mu), h \geq 0, h \neq 0$, such that $T h=h$.
\end{abstract}

\section{INTRODUCTION}

Let $(X, \Sigma, \mu)$ be a $\sigma$-finite measure space (throughout the paper, $(X, \Sigma, \mu)$ denotes a given $\sigma$-finite measure space). A measurable transformation $\phi: X \rightarrow X$ is called nonsingular if $\mu\left(\phi^{-1}(A)\right)=0$ whenever $\mu(A)=0$. A positive contraction $S: L^{\infty}(X, \Sigma, \mu) \rightarrow L^{\infty}(X, \Sigma, \mu)$ is called a Koopman operator if there exists a nonsingular transformation $\phi: X \rightarrow X$ such that $S u=u \circ \phi$ for every $u \in L^{\infty}(X, \Sigma, \mu)$. Using the Radon-Nikodym theorem it can be shown (see [5]) that every Koopman operator has a predual; that is, given a Koopman operator $S: L^{\infty}(X, \Sigma, \mu) \rightarrow$ $L^{\infty}(X, \Sigma, \mu)$, there exists a positive contraction $T: L^{1}(X, \Sigma, \mu) \rightarrow L^{1}(X, \Sigma, \mu)$ such that $T^{\prime}=S$, where $T^{\prime}$ is the dual of $T$; the operator $T$ is called a FrobeniusPerron operator (for a detailed discussion of the Frobenius-Perron operators see the book by Lasota and Mackey [5]).

Let $T: L^{1}(X, \Sigma, \mu) \rightarrow L^{1}(X, \Sigma, \mu)$ be a positive contraction.

We say that $T$ overlaps supports if for every $f, g \in L^{1}(X, \Sigma, \mu), f \geq 0, f \neq 0$, $g \geq 0, g \neq 0$, there exists $n \in \mathbb{N}(n$ depends on $f$ and $g)$ such that $T^{n} f \wedge T^{n} g \neq 0$.

If $f \in L^{1}(X, \Sigma, \mu), f \geq 0,\|f\|=1$, then it is often the custom to call $f$ a density. The operator $T$ is called (strongly) asymptotically stable if there exists a unique density $f$ such that the sequence $\left(T^{n} g\right)_{n \in \mathbb{N}}$ converges in the norm topology of $L^{1}(X, \Sigma, \mu)$ to $f$ for every density $g$ in $L^{1}(X, \Sigma, \mu)$.

If $g \in L^{1}(X, \Sigma, \mu)$, we say that $g$ is $T$-invariant (or, simply, invariant) if $T g=g$.

Received by the editors November 11, 1997 and, in revised form, January 29, 1999.

2000 Mathematics Subject Classification. Primary 47A35; Secondary 28D99, 37A30, 37A40, 47B38, 47B65. 
In 1997 Bartoszek and Brown [2] proved that if $T: L^{1}(X, \Sigma, \mu) \rightarrow L^{1}(X, \Sigma, \mu)$ is a Frobenius-Perron operator which overlaps supports and has an invariant density $f$ such that $f>0$ on $X$, then $T$ is asymptotically stable. Our goal in this note is to prove that a Frobenius-Perron operator is asymptotically stable if and only if it overlaps supports and has an invariant density.

It is of interest to point out that there exist Frobenius-Perron operators to which the Bartoszek-Brown result [2] does not apply. More precisely, it may happen that an asymptotically stable Frobenius-Perron operator has a (necessarily unique) invariant density $f$ and the set $\{f=0\}$ has strictly positive measure, as we can see from the following examples.

Example 1.1. The following example seems to be the simplest one: let $X=\{1,2\}$, let $\Sigma$ be the collection of all the subsets of $X$, let $\mu$ be the counting measure defined on $(X, \Sigma)$, and let $\phi: X \rightarrow X$ be defined by $\phi(1)=\phi(2)=1$. Then the FrobeniusPerron operator $T$ generated by $\phi$ is strongly asymptotically stable and has the invariant density $f \in L^{1}(X, \Sigma, \mu)$ defined by $f(1)=1$ and $f(2)=0$.

Example 1.2. Let $\phi: \mathbb{N} \rightarrow \mathbb{N}$ be defined by $\phi(1)=\phi(2)=1$ and $\phi(n)=n-1$ for every $n \geq 3$. If we consider the counting measure on $\mathbb{N}$, then obviously $\phi$ is nonsingular. The corresponding Frobenius-Perron operator $T: l^{1} \rightarrow l^{1}$ is defined by

$$
T\left(a_{1}, a_{2}, a_{3}, a_{4}, a_{5}, \ldots\right)=\left(a_{1}+a_{2}, a_{3}, a_{4}, a_{5}, \ldots\right)
$$

for every $\left(a_{n}\right)_{n \in \mathbb{N}} \in l^{1}$. Clearly, $T$ is strongly asymptotically stable and $(1,0,0,0, \ldots)$ is the invariant density of $T$.

Example 1.3. In the previous two examples the $L^{1}$-spaces were generated by counting measures. Let us now consider an example that involves a continuous measure. To this end, let $X=[0,2]$, let $\Sigma$ be the $\sigma$-algebra of all Lebesgue measurable subsets of $[0,2]$, let $\lambda$ be the Lebesgue measure on $[0,2]$, and let $\phi:[0,2] \rightarrow[0,2]$ be defined by $\phi(x)=2 x \bmod 1$ for $x \in[0,1]$ and $\phi(x)=x-1$ for $x \in(1,2]$. Using the example discussed on p. 116 of Horowitz [3], we conclude that if we let $T$ be the Frobenius-Perron operator corresponding to $\phi$, then $T$ is strongly asymptotically stable and $1_{[0,1]}$ is the invariant density of $T$.

Bartoszek and Brown proved (see Proposition 1 of [2]) that if $T: L^{1}(X, \Sigma, \mu) \rightarrow$ $L^{1}(X, \Sigma, \mu)$ is a positive contraction which overlaps supports, and if there exists a $T$-invariant density $g$ such that $g>0$ on $X$, then the sequence $\left(T^{n}\right)_{n \in \mathbb{N}}$ converges in the weak operator topology to a one-dimensional projection; however, the example discussed on pp. 362-364 of Akcoglu and Boivin [1] shows that, in general, $T$ is not strongly asymptotically stable. Thus, in Theorem 1 of [2] and in the main result of this note, we cannot assume that $T$ is merely a positive contraction rather than a Frobenius-Perron operator.

The paper is organized as follows: in the next section (Section 2) we discuss several lemmas which are needed in the last section (Section 3), where we prove the main result of this note.

\section{INVARIANT DENSITIES AND SUPPORT OVERLAPPING}

Let $T: L^{1}(X, \Sigma, \mu) \rightarrow L^{1}(X, \Sigma, \mu)$ be a positive contraction.

Given $A \in \Sigma$, set $\Sigma_{A}=\{B \in \Sigma \mid B \subseteq A\}$. Clearly, $\Sigma_{A}$ is a $\sigma$-algebra on $A$ and the function $\mu_{A}: \Sigma_{A} \rightarrow \mathbb{R} \cup\{+\infty\}, \mu_{A}(B)=\mu(B)$ for every $B \in \Sigma_{A}$ 
is a measure on $\left(A, \Sigma_{A}\right)$. Set also $L^{1}(A)=\left\{g \in L^{1}(X, \Sigma, \mu) \mid g 1_{A}=g\right\}$ and $L^{\infty}(A)=\left\{u \in L^{\infty}(X, \Sigma, \mu) \mid u 1_{A}=u\right\}$.

A measurable subset $A$ of $X$ is called $T$-absorbing if $T g \in L^{1}(A)$ whenever $g \in L^{1}(A)$. If $A$ is $T$-absorbing, we define an operator $T_{(A)}: L^{1}\left(A, \Sigma_{A}, \mu_{A}\right) \rightarrow$ $L^{1}\left(A, \Sigma_{A}, \mu_{A}\right)$ as follows: if $g \in L^{1}\left(A, \Sigma_{A}, \mu_{A}\right)$, then let $\tilde{g} \in L^{1}(X, \Sigma, \mu)$ be such that $\tilde{g}=g$ on $A$ and $\tilde{g}=0$ on $X \backslash A$; now set $T_{(A)} g=(T \tilde{g})_{\mid A}$, where $(T \tilde{g})_{\mid A}$ is the restriction of $T \tilde{g}$ to $A$.

Lemma 2.1. Let $\psi: X \rightarrow X$ be a nonsingular transformation, and let

$$
T: L^{1}(X, \Sigma, \mu) \rightarrow L^{1}(X, \Sigma, \mu)
$$

be the Frobenius-Perron operator corresponding to $\psi$ and to $\mu$. If $A \in \Sigma, \mu(A)>0$ is a $T$-absorbing set, then $T_{(A)}$ is also a Frobenius-Perron operator.

The proof of the above lemma follows from Theorem 1.9, p. 119 of Krengel's book [4]. Indeed, since $T^{\prime} g=g \circ \psi$ whenever $g \in L^{\infty}(X, \Sigma, \mu)$, it follows that $A$ is absorbing if and only if $\psi(A) \subseteq A \mu$ - a.e. Thus, (by possibly redefining $\psi$ on a $\mu$ negligible set) $T_{(A)}$ is a Frobenius-Perron operator corresponding to the restriction of $\psi$ to $A$.

Given a positive contraction $T: L^{1}(X, \Sigma, \mu) \rightarrow L^{1}(X, \Sigma, \mu)$ we will consider the Hopf decomposition (see Section 3.1 of [4]) of $X$ into the conservative part $C$ and the dissipative part $D=X \backslash C$ generated by $T$. We will also use the fact (see the proof of Corollary 1 of [7]) that if $T$ overlaps supports and has an invariant density, then the invariant density is unique (that is, if $T$ overlaps supports, and $f, g$ are densities such that $T f=f$ and $T g=g$, then $f=g$ ).

Lemma 2.2. Assume that $T: L^{1}(X, \Sigma, \mu) \rightarrow L^{1}(X, \Sigma, \mu)$ is a Frobenius-Perron operator which overlaps supports and has a (necessarily) unique invariant density $f$. Then $C=\{f>0\}$ and $\inf _{n \in \mathbb{N}} T^{\prime n} 1_{D}=0$, where $T^{\prime}$ is the dual of $T$.

Proof. It is well known that $\{f>0\} \subseteq C$. If we assume that $\{f>0\} \neq C$, then using the observations made on p. 126 of Krengel's book [4], and taking into consideration that $\{f>0\}$ is a $T$-absorbing set, we obtain that $C \backslash\{f>0\}$ is also $T$-absorbing. We obtain a contradiction since on one hand $\{f>0\}$ and $C \backslash\{f>0\}$ are $T$-absorbing sets, while on the other hand $T$ overlaps supports.

Clearly, in order to complete the proof of the lemma, it is enough to prove that $\sup T^{\prime n} 1_{C}=1_{X}$. To this end, note that since $T^{\prime}$ is a Koopman operator, it follows that sup $T^{\prime n} 1_{C}=1_{B}$ for some $B \in \Sigma$. Assume that $B \neq X$; that is, assume that $\mu(X \backslash \stackrel{n}{B})>0$. Then there exists $g \in L^{1}(X, \Sigma, \mu), g \geq 0, g \neq 0, g 1_{B}=0$. Since

$$
\begin{aligned}
\int\left(T^{n} f\right) \wedge\left(T^{n} g\right) d \mu & =\int\left(f \wedge\left(T^{n} g\right)\right) 1_{C} d \mu \leq \int\left(T^{n} g\right) 1_{C} d \mu \\
& \leq \int g\left(T^{\prime n} 1_{C}\right) d \mu \leq \int g 1_{B} d \mu=0
\end{aligned}
$$

for every $n \in \mathbb{N}$, we obtain a contradiction since $T$ overlaps supports.

Q.E.D.

\section{Asymptotic stability}

As mentioned in the Introduction, our goal in this section is to prove the main result of this note (Corollary 3.2 below). 
Theorem 3.1. Let $T$ be a positive contraction of $L^{1}(X, \Sigma, \mu)$ with dual $T^{\prime}$ and with conservative and dissipative parts $C$ and $D$, respectively. The following assertions are equivalent:

(a) $T$ is strongly asymptotically stable.

(b) $\inf _{n \in \mathbb{N}} T^{\prime n} 1_{D}=0$ and $T_{(C)}$ is strongly asymptotically stable.

Proof. (a) $\Rightarrow(\mathrm{b})$. Using a result of Helmberg (see Theorem 3.3, p. 175 of [4]), we obtain that $\inf _{n} T^{\prime n} 1_{D}=0$.

Clearly, the fact that $T$ is strongly asymptotically stable implies that $T_{(C)}$ is also strongly asymptotically stable.

(b) $\Rightarrow$ (a). Since $T_{(C)}$ is strongly asymptotically stable, there exists $h \in$ $L^{1}\left(C, \Sigma_{C}, \mu_{C}\right), h \geq 0,\|h\|=1$ such that $T_{(C)} h=h$. Let $f \in L^{1}(X, \Sigma, \mu)$ be defined as follows: $f=h$ on $C$ and $f=0$ on $D$.

In order to complete the proof of the theorem, it is enough to prove that $\left(T^{n} g\right)_{n \in \mathbb{N}}$ converges to $f$ in the norm topology of $L^{1}(X, \Sigma, \mu)$ whenever $g$ is a density in $L^{1}(X, \Sigma, \mu)$. Since every $u \in L^{1}(X, \Sigma, \mu)$ can be written in the form $u=u 1_{C}+$ $u 1_{D}$, and since the strong asymptotic stability of $T_{(C)}$ implies that $\left(T^{n}\left(u 1_{C}\right)\right)_{n \in \mathbb{N}}$ converges in norm to $f \cdot \int u 1_{C} d \mu$, it follows that it is actually enough to prove that $\left(T^{n} g\right)_{n}$ converges to $f$ whenever $g$ is a density in $L^{1}(X, \Sigma, \mu)$ such that $g 1_{D}=g$.

To this end, set $T_{D}=1_{D} T 1_{D}$ and note that $D$ is a strong zero set in the terminology of [8] (that is, the sequence of operators $\left(T_{D}^{n}\right)_{n \in \mathbb{N}}$ converges strongly to zero). Indeed,

$$
\int T_{D}^{n} u d \mu=\int u\left(\left(1_{D} T^{\prime} 1_{D}\right)^{n} 1_{X}\right) d \mu \leq \int u\left(T^{\prime n} 1_{D}\right) d \mu
$$

for every $n \in \mathbb{N}$ and $u \in L^{1}(X, \Sigma, \mu), u \geq 0$. Since $\left(T^{\prime n} 1_{D}\right)_{n}$ is a decreasing sequence (of elements of $L^{\infty}(X, \Sigma, \mu)$ ), and since $\inf _{n} T^{\prime n} 1_{D}=0$, it follows that $\lim _{n \rightarrow \infty}\left\|T_{D}^{n} u\right\|=0$ for every $u \in L^{1}(X, \Sigma, \mu)$.

Now, let $g$ be a density in $L^{1}(X, \Sigma, \mu)$ such that $g 1_{D}=g$, and let $\epsilon \in \mathbb{R}, \epsilon>0$. Since $D$ is a strong zero set, it follows that there exists $n_{1} \in \mathbb{N}$ such that $\left\|T_{D}^{n} g\right\|<\frac{\epsilon}{3}$ for every $n \geq n_{1}$.

By Lemma 2.2 of Lin [6] (see also Lemma 2.1 of [8]), it follows that there exist $g_{1}, g_{2}, g_{3}, \ldots, g_{n_{1}} \in L^{1}(X, \Sigma, \mu), g_{i} \geq 0, g_{i} 1_{C}=g_{i}$ for every $i=1,2,3, \ldots, n_{1}$ such that $T^{n_{1}} g=T_{D}^{n_{1}} g+\sum_{i=1}^{n_{1}} T^{n_{1}-i} g_{i}$.

Since $g_{i} 1_{C}=g_{i}$, it follows that $\lim _{n \rightarrow \infty}\left\|T^{n} g_{i}-\left(\int g_{i} d \mu\right) f\right\|=0$ for every $i=$ $1,2, \ldots, n_{1}$. Accordingly, there exists $n_{2} \in \mathbb{N}$ such that $\left\|T^{n} g_{i}-\left(\int g_{i} d \mu\right) f\right\| \leq$ $\frac{\epsilon}{3}\left\|g_{i}\right\|$ for every $n \geq n_{2}$ and $i=1,2, \ldots, n_{1}$.

Let $n_{\epsilon}=n_{1}+n_{2}$.

Since

$$
\sum_{i=1}^{n_{1}}\left\|g_{i}\right\|=\left\|\sum_{i=1}^{n_{1}} g_{i}\right\|=\left\|T^{n_{1}} g-T_{D}^{n_{1}} g\right\|=\left\|T^{n_{1}} g\right\|-\left\|T_{D}^{n_{1}} g\right\|>1-\frac{\epsilon}{3}
$$


it follows that

$$
\begin{aligned}
\left\|T^{n} g-f\right\| & =\left\|T^{n-n_{1}}\left(T_{D}^{n_{1}} g+\sum_{k=1}^{n_{1}} T^{n_{1}-k} g_{k}\right)-f\right\| \\
& \leq\left\|T^{n-n_{1}} T_{D}^{n_{1}} g-\left(1-\sum_{i=1}^{n_{1}}\left\|g_{i}\right\|\right) f\right\| \\
& +\sum_{k=1}^{n_{1}}\left\|T^{n-k} g_{k}-\left(\int g_{k} d \mu\right) f\right\| \\
& \leq\left\|T_{D}^{n_{1}} g\right\|+\left(1-\sum_{i=1}^{n_{1}}\left\|g_{i}\right\|\right)+\frac{\epsilon}{3} \sum_{i=1}^{n_{1}}\left\|g_{i}\right\| \\
& <\frac{\epsilon}{3}+\left(1-\left(1-\frac{\epsilon}{3}\right)\right)+\frac{\epsilon}{3}=\epsilon
\end{aligned}
$$

for every $n \geq n_{\epsilon}$.

Q.E.D.

Corollary 3.2. Let $T: L^{1}(X, \Sigma, \mu) \rightarrow L^{1}(X, \Sigma, \mu)$ be a Frobenius-Perron operator. The following assertions are equivalent:

(a) $T$ is strongly asymptotically stable.

(b) $T$ has an invariant density and overlaps supports.

Proof. (a) $\Rightarrow(\mathrm{b})$. If $g$ is a density, then the sequence $\left(T^{n} g\right)_{n \in \mathbb{N}}$ converges in the norm topology of $L^{1}(X, \Sigma, \mu)$ to a density $f$ which is $T$-invariant.

Let $g$ and $h$ be densities in $L^{1}(X, \Sigma, \mu)$. Since $\lim _{n \rightarrow \infty}\left\|T^{n} g-T^{n} h\right\|=0$, and since

$$
\left\|T^{m} g-T^{m} h\right\|=\left\|T^{m} g+T^{m} h-2\left(\left(T^{m} g\right) \wedge\left(T^{m} h\right)\right)\right\|
$$

for every $m \in \mathbb{N}$, it follows that there exists $n \in \mathbb{N}$ such that $\left(T^{n} g\right) \wedge\left(T^{n} h\right) \neq 0$. Thus, $T$ overlaps supports.

(b) $\Rightarrow(a)$. Let the conservative and the dissipative parts of $X$ generated by $T$ be $C$ and $D$, respectively.

Since $T$ has an invariant density, it follows that $C \neq \varnothing$.

Since $C$ is a $T$-absorbing set, Lemma 2.1 tells us that $T_{(C)}$ is a Frobenius-Perron operator, while Lemma 2.2 implies that $T_{(C)}$ satisfies the conditions of Theorem 1 of [2]; hence $T_{(C)}$ is strongly asymptotically stable.

Finally, using Lemma 2.2 and Theorem 3.1 we conclude that $T$ is strongly asymptotically stable.

Q.E.D.

\section{ACKNOWLEDGMENT}

We thank the anonymous referee for various recommendations which have improved the exposition significantly.

\section{REFERENCES}

1. M.A. Akcoglu and D. Boivin, Approximation of $L_{p}$-contractions by isometries, Canad. Math. Bull. 32 (1989), 360-364. MR 91b:47018

2. W. Bartoszek and T. Brown, On Frobenius-Perron operators which overlap supports, Bull. Polish Acad. Sci. Math. 45 (1997), 17-24. MR 98i:47006

3. S. Horowitz, Some limit theorems for Markov processes, Israel J. Math. 6 (1968), 107-118. MR 39:4930

4. U. Krengel, Ergodic Theorems, Walter de Gruyter, Berlin, 1985. MR 87i:28001 
5. A. Lasota and M.C. Mackey, Chaos, Fractals and Noise, Springer, New York, 1994. MR 94j:58102

6. M. Lin, Convergence of the iterates of a Markov operator, Z. Wahrscheinlichkeitstheorie verw. Geb. 29 (1974), 153-163. MR 51:1966]

7. R. Rudnicki, On asymptotic stability and sweeping for Markov operators, Bull. Polish Acad. Sci. Math. 43 (1995), 245-262. MR 97m:47041

8. R. Zaharopol, A local zero-two law and some applications, submitted.

Department of Mathematical Sciences, Binghamton University (S.U.N.Y. at BingHAMton), Binghamton, New York 13902-6000

E-mail address: radu@math.binghamton.edu 